\title{
Application of Mind Mapping in the Course Construction of the Technology of Aviation Datalink
}

\author{
Juan Chen", Lei Zhang, Xueqiang Zheng \\ College of Communication Engineering, Amy Engineering University of PLA, Nanjing, China \\ Email address: \\ pumpkin0910@163.com (Juan Chen), leizhang@163.com (Lei Zhang), zxqq302@163.com (Xueqiang Zheng) \\ ${ }^{*}$ Corresponding author
}

To cite this article:

Juan Chen, Lei Zhang, Xueqiang Zheng. Application of Mind Mapping in the Course Construction of the Technology of Aviation Datalink. Science Discovery. Vol. 6, No. 5, 2018, pp. 414-420. doi: 10.11648/j.sd.20180605.26

Received: September 17, 2018; Accepted: October 29, 2018; Published: November 8, 2018

\begin{abstract}
In early 1960s, British "Mr. Brain" Tony Buzan has fathered a creative tool-Mind Map, which soon became popular and was occupied widely in many fields, especially in the field of education. Mind Mapping which improves intelligence and raises the level of thinking, has been recognized by more and more people. Mind maps help people learn information through organizing it and adding images and coloring to it. With the demonstration of its practice effect, Mind Map's potential value has attractived some relevant personages in Chinese education circle. Mind mapping is a visual way to record and organize information where a "map" is drawn up which does not rely on large amounts of written information, but on textual descriptors and graphical clues. As a visualization tool, Mind Mapping provides a strong support in optimaizing the teaching activities and the course construction, which is an important content of teaching basic construction in colleges and universities. Strengthening the course construction is effective to carry out the teaching plan, lift the level of instruction and the talent training quality. The foundation of the course construction is the textbook construction. Because of the lack of corresponding textbook in the course of the Technology of Aviation Datalink, it needs to write a new suitable textbook. Through analyzing of the difficulties of textbook compilation, it emphasizes the necessity of Mind Mapping. With using Mind Mapping in the textbook construction, it improves the process of the textbook compilation. Writing the textbook is clear, scientific and efficient, and the textbook is logical and comprehensible. Moreover, using Mind Mapping can enhance the relationship of the editors. In this paper, it uses the MindManager software, and presents some operational software skills.
\end{abstract}

Keywords: Mind Map, Course Construction, Aviation Datalink

\section{思维导图在《航空数据链技术》课程建设中的应用研究}

陈娟", 张磊, 郑学强

通信工程学院, 陆军工程大学, 南京, 中国

邮箱

pumpkin0910@163.com (陈娟)，leizhang@163.com（张否），zxqq302@163.com（郑学强）

摘要: 20 世纪60年代初期, 英国“大脑先生”东尼·博赞始创了创造性思维工具——思维导图, 目前, 思维导图已经在学 习、工作等多个领域广泛应用, 特别是教学领域, 用思维导图提升智力能力、提高思维水平, 已得到越来越多人的认 可。伴随着思维导图应用效果的凸显, 其潜在的教育价值已经引起了教育界人士的热切关注。作为一种可视化的工具, 思维导图在优化教学活动、促进课程建设方面提供了强有力的支持。课程建设是高等院校教学基本建设的重要内容之 一, 加强课程建设是有效落实教学计划、提高教学水平和人才培养质量的重要保证，其中，教材建设是基础。由于缺 乏适用于《航空数据链技术》课程的教材, 迫切需要采取自编的方式解决问题。本文在撰写教材过程中引入思维导图 工具, 改善了传统教材编写中的诸多问题, 更好地理清了思路, 加深了对知识点的理解, 使教材编写过程科学、高效, 
且教材结构完整、逻辑性强、条理清晰, 同时, 增强了编者之间的沟通交流。文中还给出了部分软件使用技巧, 具有 可操作性。

关键词: 思维导图, 课程建设, 航空数据链

\section{1. 引言}

课程建设, 从广义方面来讲, 是指形成和决定课程质 量的各种条件和内涵建设, 如教学理论建设、师资队伍建 设、环境建设、教学资源建设等。从狭义方面来讲，课程 建设是指以某一学科或某一专业的培养目标为核心的课 程体系建设以及该体系中具体课程的建设。课程建设是一 所学校教学的本质需求, 从功效的角度看, 加强课程建设 是有效落实教学计划的重要保证, 是稳定和提高教学水平、 教学质量的重要手段, 是一个学校可持续发展的依据[1]。 对具体的某课程而言, 课程建设包含选取授课教材、设立 课程标准、编拟实施计划、确立教学模式、教学手段、教 学方法等, 其中选择适用于课程的教材是基础。

本学期针对无人机专业学生开设了一门新课程《航空数据链技术》, 但是在初期教材的选择上面临了诸 多困难。首先, 无现成的针对性教材, 目前可供选择的相 关书籍大多是从战术数据链的角度进行描述, 如骆光明的 《数据链一一信息系统链接武器系统的捷径》、赵志勇的 《数据链系统与技术》等; 其次, 对无人机数据链进行原 理叙述的教材少, 能找到的参考材料基本为操作手册, 无 法针对学生进行有的放矢地教学。介于此, 课题组须采用 自编教材的方式来解决问题。

虽然在理论界有很多博士、硕士学位论文都对航空数 据链进行了介绍分析, 如电子科技大学张否同学的《航空 数据链组网技术研究》、汕头大学林春同学的《基于 $1090 \mathrm{ES}$ 的ADS-B的应用分析与研究》等, 但不同的专家学者来自 各个专业领域, 所研究的内容侧重点不同, 导致对数据链 的定义和理解不尽相同, 如何抓住本质、分析问题、梳理 脉络, 确立统一的数据链定义方式, 难度非常大。

要充分挖掘现有和潜在的资源, 并合理运用, 课题组 在撰写教材过程中引入了近年来在诸多领域广泛应用的 思维导图, 用其来分析资料、整理概念、建立纲目、撰写 教材, 取得了良好的成效。本文以编写教材为切入点, 分 析了思维导图在《航空数据链技术》课程建设中的应用, 展示了其在该领域的可操作性和有效性。

\section{2. 思维导图简介}

“思维导图是一种可视图表,一种整体思维工具, 可 以应用到所有认知功能领域, 尤其是记忆、创造、学习和 各种形式的思考。[2]”目前, 思维导图已经在各领域广泛 应用, 特别是教学领域。哈佛大学和剑桥大学的师生都在 使用思维导图这项思维工具进行教学和学习。在新加坡, 思维导图已成为中小学生的必修课。用思维导图提升智力 能力、提高思维水平, 已得到越来越多人的认可。世界 500 强的众多公司, 更是把思维导图课程列为员工进入公司的
必修课[3]。而在国内其尚处于引进与发展阶段。伴随着思 维导图应用效果的凸显, 其潜在的教育价值已经引起了我 国教育界部分相关人士的热切关注 [4]。作为知识可视化的 工具, 思维导图以文字和图像相结合的形式, 将抽象思维 与形象思维有机结合, 为优化教学活动提供了新视角和技 术支持 [5], 影响着人们对文本、思维想象的再认识, 使我 们的教学活动成为一种创造性的思维盛会[6]。

\section{1. 思维导图的概念}

思维导图（Mind Map）是由世界创造力智商最高保 持者东尼・博赞（Tony Buzan）先生于20世纪60年代发明 创造的一种便捷思维工具, 它将放射性思考具体化, 自然 体现了人类大脑的功能。思维导图以图文并茂的形式和网 状脉络的结构, 储存、组织、优化和输出信息, 能提高人 类思考的效率, 提升记忆力和逻辑力, 并增进创造力。思, 用心思考; 维, 多维度立体化; 导, 信息的输入和输出; 图, 图解方式体现[7]。

人的大脑分左右两个半球, 左半球负责逻辑思维, 像 一位科学家, 处理如词汇、运算、顺序等内容, 而右大脑 负责抽象思维, 像一位艺术家, 处理如图像、音乐、语言 和美术等内容。相对于各类繁复的信息, 人的大脑更容易 记住直观的东西, 比如数字、图形、气味、颜色、节奏等 [1]。如何将复杂的问题简单化, 找到各个关键点并寻求相 互之间的联系, 看清本质, 是思维导图的主要功能, 它将 逻辑思维和抽象思维结合起来, 让大脑两个半球相互协作, 相辅相成, 使其尽可能发挥出所有的潜能。东尼・博赞认 为, “思维导图是对发散性思维的表达, 是一种打开大脑 潜能力的万能钥匙”[8]。

\section{2. 思维导图的基本特征}

思维导图使用一个中央关键词, 以多条辐射线连接多 个代表想法、任务、概念的相关词语, 并以图式结构描述 这些词语的关联，建立一种非线性式、以辐射线形方式向 多个方向扩展的连接。这些连接有些是逻辑的, 有些是自 由的, 根据不同的任务而有所不同 [9]。东尼・博赞在其著 作中, 认为思维导图有四个基本特征: “其一, 注意的焦 点清晰地集中在中央图形上。其二, 主题的主干作为分支 从中央向四周放射。其三, 分支由一个关键的图形或者写 在产生联想的线条上面的关键词构成。比较不重要的话题 也分支形式表现出来, 附在较高层次的分支上。其四, 各 分支形成一个连接的节点结构, 因此思维导图在表现形式 上是树化的。”[4]

思维导图概念传入我国后, 我国学者根据国人认知特 点及理解能力, 重新划分思维导图的表现形式。华东师范 大学现代教育技术研究所刘濯源将思维导图划分为归纳 型、分析型和创造型三种方式。刘濯源认为, “思维导图 
可依据教学目的分为三种类型, 用于对知识进行归纳总结 的被称为归纳型思维导图; 用于题目或文章分析的被称为 分析型思维导图; 帮我们快速打开思路、进行结构化发散 思考的则被称为创作型思维导图。”[10]

\section{3. 思维导图软件工具}

思维导图的绘制分为手绘和软件绘图, 两种方式各有 利弊。使用纸笔手绘更加自由, 可以不受束缚把内心的思 考较为全面地表达出来, 但是在出现错误时不便纠正, 在 构图上也会有不尽人意之处, 常常需要重复绘制才能达到 最佳效果; 软件绘图, 更适合工作处理数字化的需求, 便 于编辑和加工, 在信息传播中也更为便捷, 其绘制的思维 导图质量和美观程度均有所提高。因此, 在实际工作中, 思维导图软件使用更为广泛[3]。

前期作者对多种软件，如Xmind、MindManager、幕 布、百度脑图等, 进行了试用与对比, 最终选择了装机率 最高、应用最为广泛的思维导图软件 MindManager。 MindManager由Mindjet公司开发, 软件应用分为头脑风暴、
项目管理和会议管理三个方面, 诸多著名的跨国企业都是 其客户。无论从功能性还是软件设计成熟度上, 该软件工 具表现非常出色, 可以满足用户的基本需求, 适合做大型 的思维导图。其特点为:

（1）用户界面设计友好, 功能丰富, 能较好地提高 项目组的工作效率和小组成员之间的协作性;

（2）主题创建简单，多个快捷键配合使用，操作性 好;

（3）素材库丰富, 包括基本图形、数字、人物等多 个领域的彩色静态、动态图标, 使思维导图展现得更为生 动, 增强学习效果;

(4) 超链接应用广泛, 主题或文字均可使用超链接, 只要相对路径保持不变, 相关的资料均可使用链接加入到 导图中;

(5) 与Office软件的整合和互操作性较好, 可导出到 Word、PowerPoint, 还可以直接在MindManager中选择PPT 模板, 进行分块演示, 便于汇报和集体讨论。[9][11]

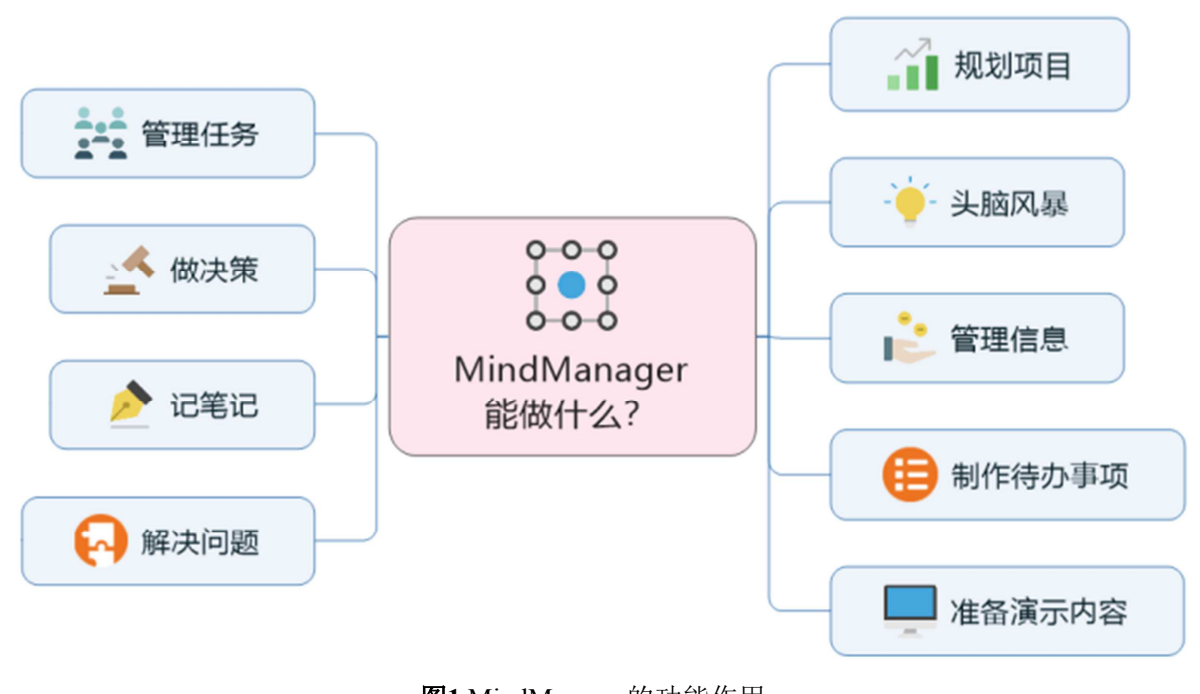

图1 MindManager的功能作用。

\section{3. 传统教材编写方法的不足}

在传统的教材编写流程中, 一般采取的方法是使用文 档编辑软件, 线性式列出书籍纲目, 根据需求查找资料、 补充内容, 再经过讨论修改形成终稿。在这个过程中, 主 要有以下不足:

（1）把握整体布局困难。条目式的教材框架不能很 好地体现出章节间的相互联系, 观点、概念的条理性不够 清晰。

（2）整理文献资料困难。搜索各类资料简单，但是 进行归类分析难。仅依靠记录资料大概内容、主要观点及 出处, 在后期查阅时难以根据需要快速查找; 另外, 专业 术语的统一度欠缺。

（3）编者沟通交流困难。如果参与教材编写的人员 较多, 其所撰写的内容多有重复或者冲突, 在讨论时难以 把握重点, 区分主次。

在使用思维导图之后, 以上困难均会有所缓解。

\section{4. 思维导图在教材编写中的应用}

\section{1. 分析需求, 建立纲目}

思维导图是实现头脑风暴过程的完美工具, 因为参与 会议的每个人其能力都非常重要, 通过创建和共享讨论内 容的视觉导图, 每个人都能看到自己提出的建议所产生的 影响和优先级决策, 现场会议结束后, 导图便可立即发布 出来, 无需参会者再抄写笔记。MindManager多选的导图 格式, 还可以提供显示重点的灵活性, 在单一视图中深入 地显示细节。因此, 在分析需求阶段就可以应用思维导图。

首先, 组织教学小组集中讨论, 通过教学目标的确立 和对教学对象的分析, 提出教学需求, 确定教材基本内容, 并确立教材名称为《航空数据链概论》。各成员围绕教材 展开讨论并记录商议内容, 各抒已见的同时, 把 MindManager当成手中的虚拟白板, 以书名为中心主题建 立思维导图, 这样有利于界定该主题的核心理念, 让所有 
的讨论内容均能够紧紧围绕中心主题而不偏离, 把控讨论 的重点。轻松快速地将每个人的想法收集并展示, 尽可能 用浮动主题将讨论时所提及到的所有内容添加到页面上, 聚焦每个人流动的思维。

其次, 经过汇合与统一, 建立一个辐射状导图。在中 心主题上, 顺时针方向添加各章节内容作为二级中心主题, 形成核心主轴思考[5], 确立初步框架。通过思维导图, 可
以帮助参与者查看概念间的关联以及如何适应工作范围。 为了能直观体现教材框架的层级, 可以对思维导图的多级 中心主题增加自动编号。在对某章节讨论时提及的不可或 缺的内容, 可以及时添加下一级主题。同时, 为防止遗漏 相关讨论细节，可以在对应主题上增加备注或“便签”，便 于后期查阅。

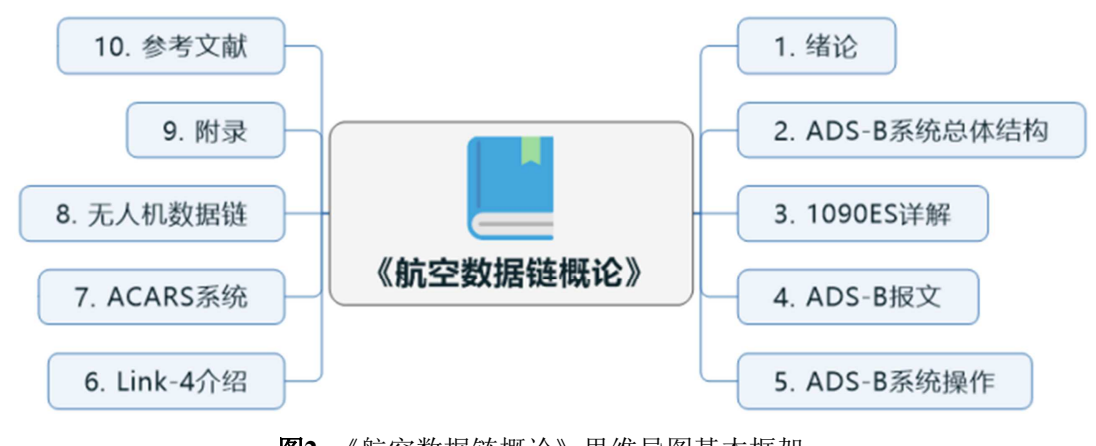

图2《航空数据链概论》思维导图基本框架。

再次, 建立教材初步框架后, 根据会议讨论情况制作 一份工作计划思维导图, 将教材编写的进度安排、人员责 任分工情况、章节内容的特殊情况说明、关键词的名称统 一、值得关注的重点问题等, 集中体现出来, 让每一名编 者都能通过查看思维导图, 掌控教材编写的整体进展, 明 确任务, 目标集中, 重点突出, 做到心中有数。

在制作完编书工作计划的思维导图后，可以根据每一 个主题的内容, 添加合适的能让人产生联想的小图标, 可
视化的特殊标记生动形象, 让人一目了然, 印象深刻。如 中心主题“编书工作计划”, 使用多个人物在一起的图标, 表示多人协作；“进度”主题用钟表图标，表示时间进程; “责任分工”主题使用工具图标, 表示每个人要完成的任务; “关键词统一”主题使用工字图钉图标, 表示固定词语; “重 点关注” 使用放大镜图标, 表示需要特别注意到的内容。

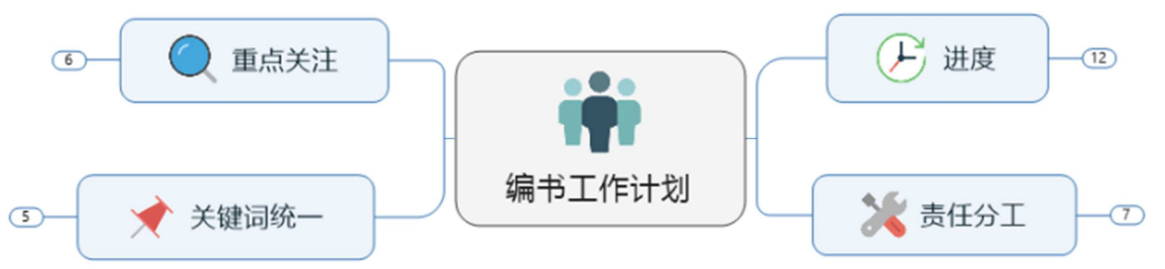

图3《航空数据链概论》编书工作计划思维导图。

\section{2. 准备资料, 整合内容}

资料准备，不仅包括收集各种资料文献，还包括对资 料进行研究、分析和篮选。

在确立教材基本框架之后, 充分利用各类数据知识服 务平台, 以关键词广泛获取学术资源, 收集文献资料。经 初步汶览后, 将各个数据库中检索到的文献, 根据内容分 门别类进行归并, 建立资料包。另外, 还要收集与拟编教 材类别相同的教材, 尽可能全面研究和分析它们, 扬长避 短, 使自编教材满足教学需求。

在纷繁复杂的资料面前, 琐碎的内容和千丝万缕的关 系不能直观体现, 如果全凭记忆来选取可用内容几乎是不 可实现的。如 《ADS-B监视功能的性能研究和仿真》和 《1090ES ADS-B接收机嵌入式软件设计》等多篇文献中 都提及了ADS-B数据链的三种标准, 但是侧重点和对比角 度不同, 如果是只记录概要, 则无法区分两篇文献的不同 之处, 在选取参考对象时就难以取舍。因此, 如何分析文 献、整合内容成为了一个亟待解决的问题。作为 MindManager最基本的功能之一, 整理文章内容是分析型
思维导图的优势所在。因为思维导图本身是中心发散层级 结构, 这种结构与一篇文章的内容结构是有相似之处的, 导图中心相当于文章中心, 导图分支相当于文章各关键点, 导图的层次分支相当于文章的具体细节内容 [12]。

分组建立资料包后, 通过泛读挑选出数篇重点文献, 分别进行内容整理。以文献名称为中心主题, 对编者重点 关注的章节和内容进行分析, 以多层级方式展现出关键点 和细节。此时, 一张导图就可以体现一篇文献的概况和主 要内容, 层次分明、思路清晰且直观易懂。在分析完文献 后, 组织教学小组二次集中讨论, 除分享文献的整理情况 外, 更重要的工作, 是对不同文献中提及到的相似概念及 数据链定义方式进行分析讨论, 确立统一的名称、解释及 分类方法, 使编写的教材内容前后一致, 然后将思维导图 中的必要内容与前期拟制的纲目进行整合。

在思维导图中需要重点关注的地方, 可以使用图标、 颜色、标记等手段，以吸引编者的目光[13]。同时可以增 加必要的便签、链接等, 以便于查看文字、图片说明及对 应文献, 避免来源不清。 


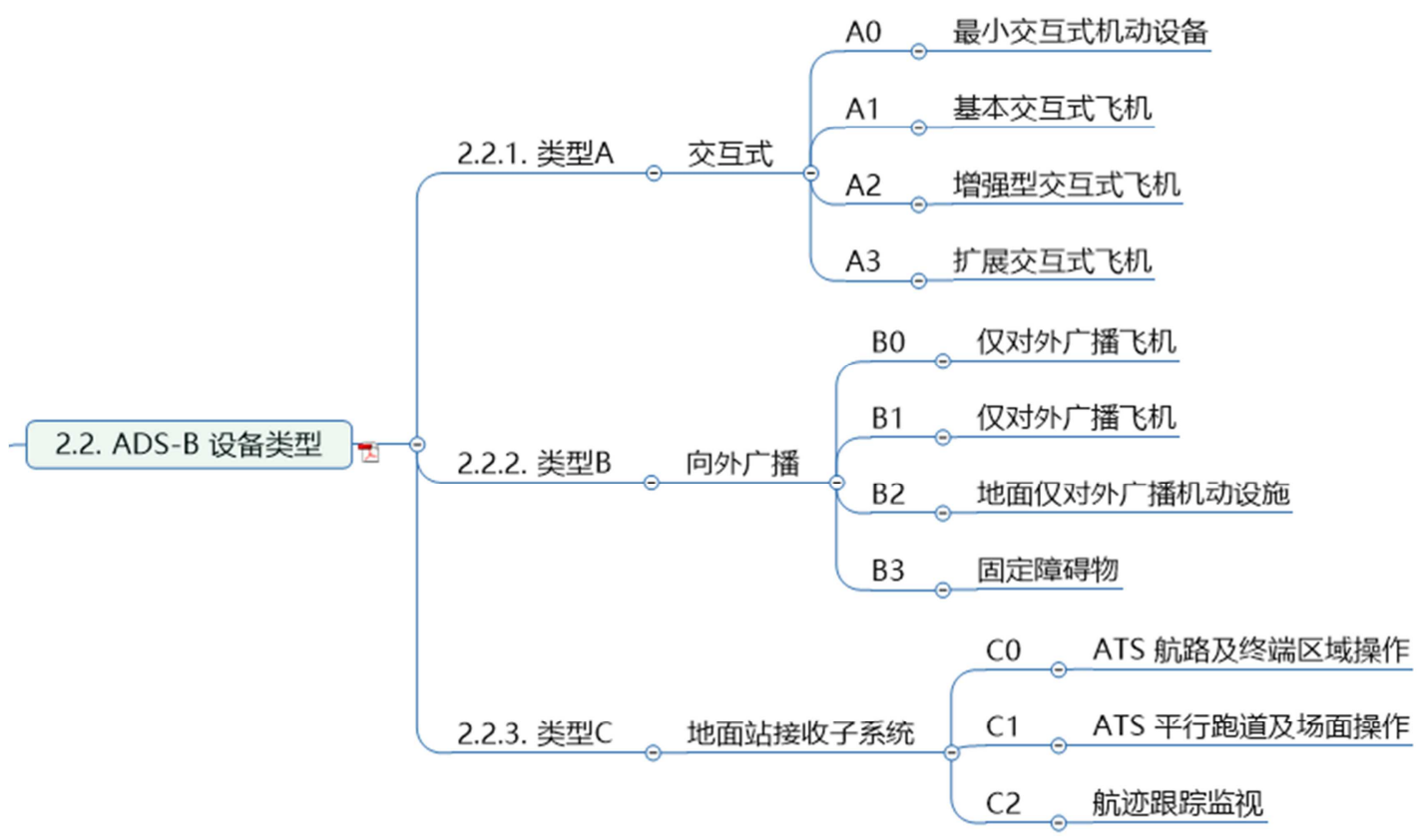

图4《航空数据链概论》思维导图局部内容。

教材思维导图的纲目全部整理完毕后, 为了便于在教 学小组讨论时向组员展示导图内容, 使成员能够清晰地看 到每一中心主题的细节, 可以使用MindManager的“演示文 稿”功能。该功能可以自动生成幻灯片, 在幻灯片播放状 态下也允许随时编辑修改思维导图的内容。

\section{3. 导出大纲, 编写初稿}

确定纲目细节后, 就可以把思维导图导出为需要的 文件类型, 如汶览器中可以查看的交互式只读导图、图 像文件、电子表单、Word文档、PowerPoint演示文稿等 等。这里, 我们使用的是Word文档。在“导出设置”中, 可以对文档的模板、编号、链接显示、目录等常规信息 进行设置。在分析需求阶段, 我们已经设置了级别的编 号, 其对应了Word文档中各级标题格式, 导出的文档以 大纲形式展现。因此, 只要前期整理的教材思维导图脉 络清晰, Word文档就会结构完整、级别统一。且在思维 导图的各个主题中添加的“链接”、“标签”等内容也都在 文档中体现出来, 相关的文献信息以超链接的方式在对 应章节显示, 编写教材时能直接通过超链接打开参考文 献, 减少了重新查找文献所耗费的时间和精力。此时, 可以向参与教材编写的各位编者分享教材思维导图、 Word文档以及参考文献资料, 根据前期分工和时间节点, 各编者进行相关章节的编写工作，尔后，由主编进行合 稿, 形成教材初稿。

\section{4. 完善细节, 形成终稿}

初稿完成后, 主编组织编者对编写情况进行讨论, 将 编写过程中遇到的问题、新的见解、考虑欠缺的地方进行 商议和补充, 同时进一步更新教材思维导图, 根据需求查 找文献, 并在此基础上对教材内容进行修订。经过多次更 新和完善后, 教材内容基本上趋于完整, 最后的工作则是 校对和出版。

\section{5. 软件使用技巧}

在教材编写的起始阶段, 我们的重心放在前期思维导 图的规划上, 因为没有科学、完整的规划, 就容易失去方 向, 思维凌乱, 也就不可能写出一本结构合理、内容充实 的教材。为了更好地完成这个规划, 我们利用了 MindManager软件的多种功能, 这里, 将软件的部分使用 技巧做一说明。

（1）添加多级序号。单击中心主题, 找到“插入”选 项卡中“编号”，在其下拉菜单中选择“编号选项”，则可以 定义编号方案、编号深度、标签等, 使该主题下的所有副 主题按深度形成多级编号, 深度根据层级递进, 可以设定 的级别数量最多为 5 级。设置完毕后可以自动生成编号。 在导出Word文档时, 直接形成多级标题。 


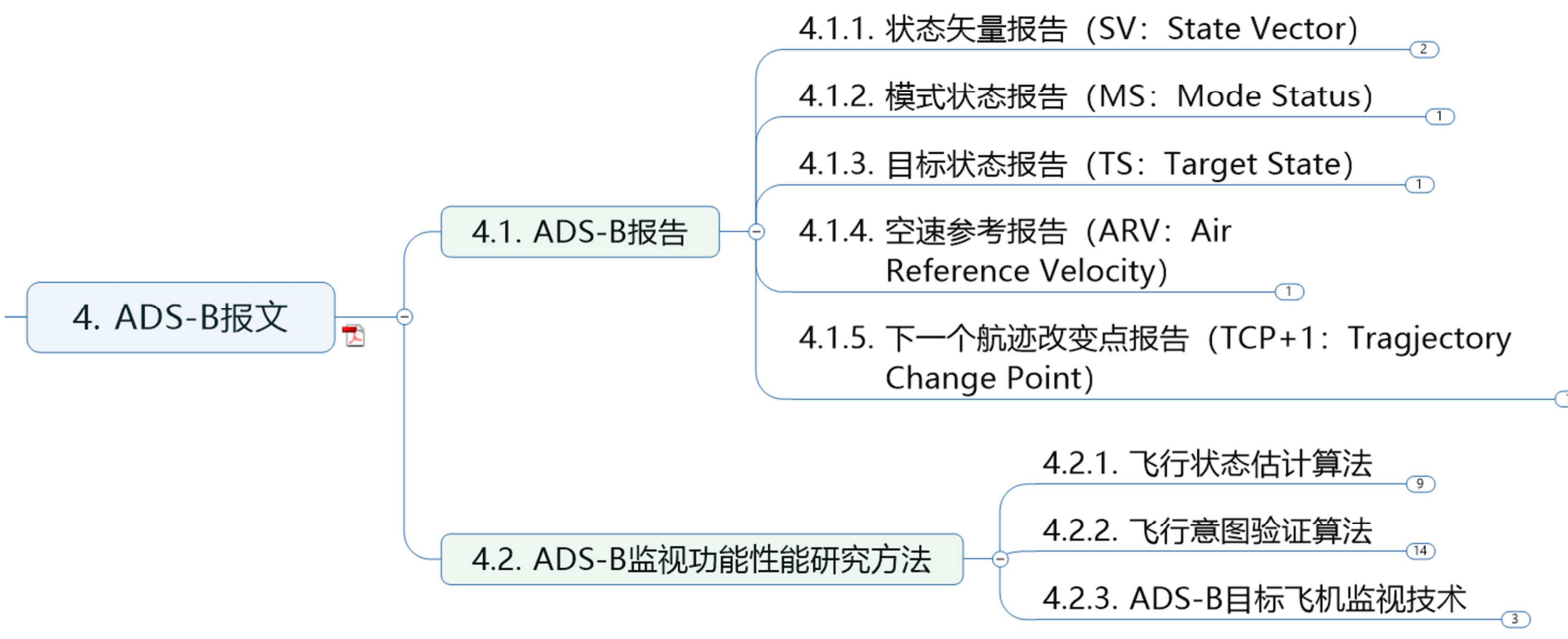

图5 在思维导图中添加“编号”。

(2) 添加链接。在MindManager中, 可以为某一主题添加链接, 主要有三种: URL/文件路径、此导图中的主题、 电子邮件地址。“主页”选项卡下找到“主题元素”模块，单击“链接”，则可以添加不同的链接类型。在编写教材中，用 得较多的是文件链接, 可以使编者便捷快速地打开相关文献, 从而避免出现找不到内容对应参考文献的问题, 提高了 查找文献的效率。

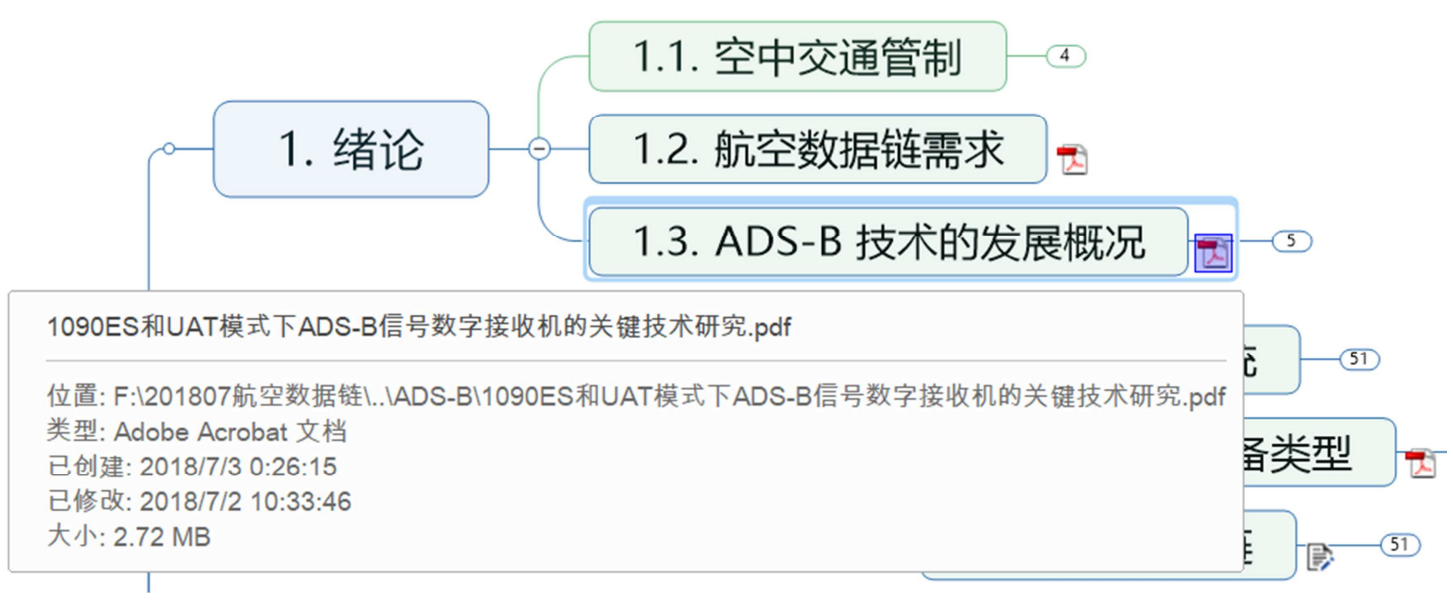

图6 在思维导图中添加“链接”。

（3）添加便签。如果对某一主题有较多的文字、图入内容，除文字信息外，还可以插入表格、链接、图片、 片说明, 或者需要使用的链接较多时, 可以使用便签功能。 日期和时间等，可以完整地向编者传递说明信息。

找到“插入”选项卡中“便签”，在打开的主题便签页面上输

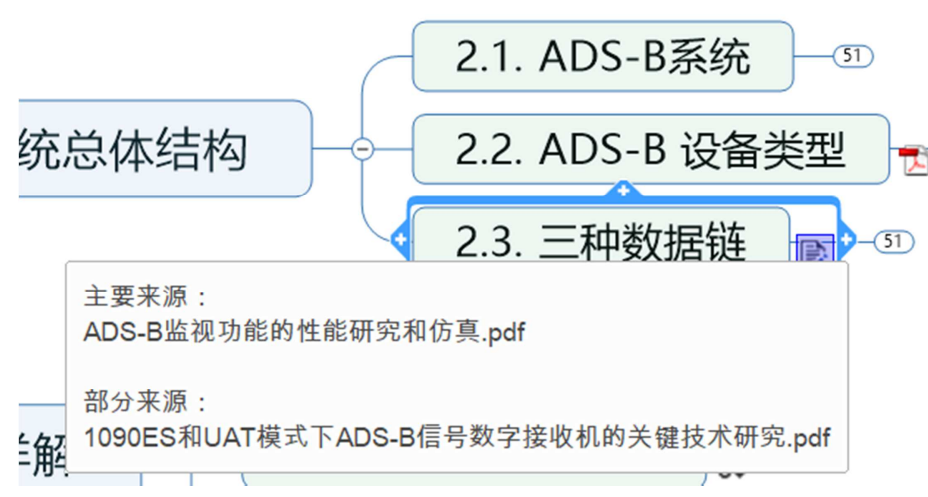

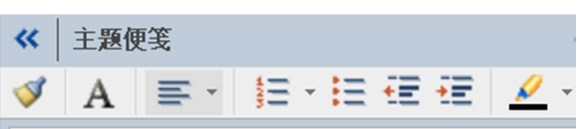

三种数据链

主要来源:

ADS-B监视功能的性能研究和仿真.pdf

部分来源:

1090ES和UAT模式下ADS-B信号数字接出

图7 在思维导图中添加“便签”。 
（4）演示文稿。在集体讨论过程中需要即时有效地 向与会者展示思维导图细节时, 演示文稿功能就可以大展 身手了。在“视图”选项卡中选择“演示”, 自动创建幻灯片, 则将每一个二级主题及其包含的内容生成一张幻灯, 然后 选择自动播放幻灯。与PowerPoint不同的是, MindManager 在幻灯播放过程中，可以放大、缩小，对里面的内容进行 修改, 增加便签、链接、图片等操作, 即演示文稿具有与 导图视图下同等的功能。这样可以在展示内容的同时, 根 据与会者的反馈随时修改思维导图。

\section{6. 结论}

发挥课程在人才培养中的重要作用, 就要科学推进课 程教材建设。一本符合课程标准、利于实现培养目标的教 材, 是课程建设的重要载体。在撰写教材过程中引入思维 导图工具, 改善了传统教材编写中的诸多问题, 不仅有利 于让编者形成发散思维, 产生新想法, 还能理清思路, 加 深对知识点的理解, 使教材编写过程科学、高效, 也有利 于撰写结构完整、逻辑性强、条理清晰、系统、先进的教 材 [14], 进而为学生夯筑坚实的专业基础、培养拔尖的专 业能力、引导流畅的思维方式。思维导图能增强编者之间 的沟通交流, 充分发挥个人创造力, 激活个人潜力, 使编 者纵览全局, 更加有效地规划部署工作并有力执行。院校 课程建设任重道远, 如何更加高效利用思维导图工具, 提 高教学质量过程, 还需要进一步研究与实践, 以获取更加 广阔的发展空间。

\section{参考文献}

[1] 杨晓辉, 高校课程建设思维模式与方法探索, 天津中德应 用技术大学学报 $[M], 2018$ 年4月第2期。
[2] 程锐, 思维导图在高校课程教学中的应用研究, 软件导 刊·教育技术 $[\mathrm{M}] ， 2015$ 年11期。

[3] 思维导图在高校教学中的应用研究: 过程与模式 [J], 于立生, 海峡科学，2015年第2期（总第98期）。

[4] 郭艳霞, 基于思维导图的教学模式研究[D], 湖南师范大学, 2011年05月。

[5] 于承敏, 王丽雁, 基于思维导图的《计算机网络》课堂教 学研究, 电脑与电信 $[\mathrm{M}], 2018$ 年第 1 期。

[6] 刘晓宁, 我国思维导图研究综述[M], 四川教育学院学报, 第 25 卷第 5 期, 2009 年 5 月。

[7] 刘艳, 你一学就会的思维导图[M], 文化发展出版社, 2017 年4月，ISBN：978-7-5142-1654-7。

[8] 刘良华, 教育研究方法专题与案例 $[M]$, 上海: 华东师范大 学出版社, 2007年。

[9] 华晓宇, 陈国明, 应用视角下思维导图软件的比较与选用 策略 $[\mathrm{J}]$ ，现代教育技术，2016年第26卷第 1 期。

[10] 杨蒙, 思维导图对遗忘知识再学习效果的实验研究 [D], 天 津师范大学, 2017年5月。

[11] 赵娜, 浅谈Mind Manager软件在高校课堂中的作用一以 《思想道德修养与法律基础》第一章为例, Computer CD Software and Applications[M]，2014年第3期

[12] 整理资料为何不用 MindManager 思维导图 [Z] , http://www.mindmanager.cc/rumenjiaocheng/, 2016-02-15.

[13] The Effects of Mind Mapping Activities on Students' Motivation, Brett D. Jon, Chloe Ruff, Jennifer Dee Snyder, Published by Digital Commons@Georgia Southern [M], 2012.

[14] 杜学允, 思维导图在创新型人才培养中的应用研究[D], 哈 尔滨理工大学，2013年03月。 